\title{
BISTATIC SAR ALONG TRACK INTERFEROMETRY WITH MULTIPLE FIXED RECEIVERS
}

\author{
Sergi Duque ${ }^{(1)}$, Paco López-Dekker ${ }^{(1)(2)}$, Juan C. Merlano ${ }^{(1)}$, and Jordi J. Mallorqui ${ }^{(1)}$ \\ ${ }^{(1)}$ Remote Sensing Laboratory (RSLab) - UPC, Spain \\ ${ }^{(2)}$ DLR, Germany
}

\begin{abstract}
This paper presents an along - track interferometry (ATI) study for a bistatic or multiestatic SAR configuration with fixed ground receivers. This technique can be useful for sea current estimation or for any problem of Ground Motion Target Indicator (GMTI). The proximity of the ground receivers to the scene allows to be very sensitivite to velocities with small baselines. This paper also proposes a multibaseline approach for ATI able to diferenciate among different velocity contributions in the same resolution cell. At the end of this paper, some results over real acquired bistatic data will be presented and discussed. The data have been acquired using the C-band SAR Bistatic Receiver for INterferometric Applications (SABRINA) and ESA's ENVISAT satellite, as a transmitter of opportunity.
\end{abstract}

Index Terms - SAR, Biestatic, ATI, Multibaseline

\section{INTRODUCTION}

Although Bistatic SAR (BSAR) systems have been studied for the first time more than 25 years ago, they have become popular over the last decade. The scientific interest about BSAR has been focused on the image formation and on system aspects, such as phase synchronization between transmitter and receiver [1]. Anyway, the interest in BSAR should be moved to the applications side. It has to be highlighted that in a BSAR or in a Multistatic SAR (MSAR) configuration, only one transmitter is needed and multiple transmitters can be freely deployed allowing to observe the scene from different points of view. In addition, the receivers are cheaper then their monostatic counterparts that have to include also a transmitter.

The Remote Sensing Lab (RSLab) at the Universitat Politècnica de Catalunya is studying interferometric applications of fixed-receiver BSAR systems [2]. This configuration presents two important advantages in interferometric and

This work has been supported by the Spanish MCYT funds under the projects TEC2008-06764-C02-01 and TEC2007-65690/TCM, the Spanish Ramon y Cajal program, the Catalan Commission for Research (CIRIT) and the Spanish MEC granting the first author (AP2005-1484).

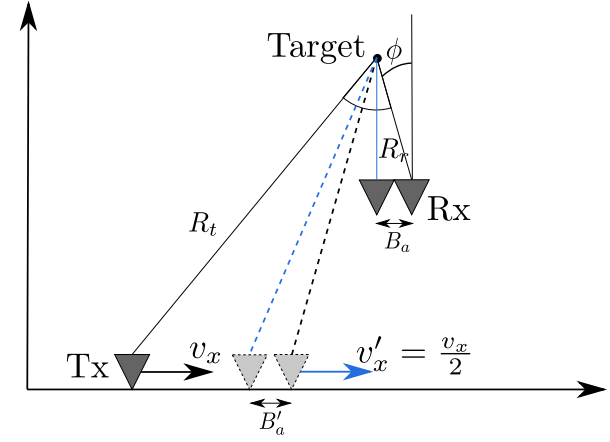

Fig. 1. ATI bistatic geometry. The figure shows a single moving transmitter, two fixed receivers with an along-track baseline, and the equivalent monostatic phase-centers forming a moving along-track pair.

tomographic applications. The first one, is that due to the relative proximity of the receiver to the region of interest, good interferometric results can be produed with short baselines on the receive end. The other is that the acquisition can be done in a single-pass configuration, elliminating the temporal decorrelation.

This paper studies BSAR Along-Track Interferometry (ATI) using multiple fixed-receivers an a satellite as a transmitter. The theoretical development of BSAR-ATI is complemented wiht the experimental results obtained with SABRINA-C and a target with controlled velocity.

\section{BSAR ATI THEORY}

\subsection{Monostatic equivalent}

Figure 1 illustrates the BSAR ATI geometry considered in this work. A single transmitter moves at an effective velocity $v_{x}$ illuminating the target. The scattered signal is received by two (or more) antennas separated by an along-track baseline, $B_{a}$. The geometry can be considered as an equivalent monostatic system with a set of antennas placed along the bisectors of the angles formed by the transmitter antenna, the target, and the receivers. These bisectors rotate as the transmitter follows its trajectory with an angular velocity that is 
half that of the monostatic one, $\omega_{t}^{\prime}=\omega_{t} / 2$, being the monostatic $\omega_{t}=v_{x} / R_{t}$. Thus, the monostatic equivalent effective velocity is

$$
v_{x}^{\prime}=\frac{v_{x}}{2} .
$$

The along-track baseline in this case is given by

$$
B_{a}^{\prime}=\frac{R_{t x}}{2 R_{r x}} B_{a},
$$

where $R_{t x}$ and $R_{r x}$ are the transmitter-target range and the receiver-target range respectively. Thus, for an orbital transmitter in combination with a ground-based receiver, a short receiver baseline corresponds to orders of magnitude than the equivalent orbital one. The ATI temporal lag can be obtained by dividing (2) by (1),

$$
\tau_{A T I}=\frac{B_{a}}{v_{x}} \frac{R_{t}}{R_{r}}=\frac{B_{a}}{\omega_{t} R_{r}} .
$$

Taking a look to Figure 1 it can be deduced that there is an interferometric phase component associated to the angular position, $\phi$, of the target with respect to the ATI baseline. In fact, if there is no moving target, the ATI phase can be expressed as

$$
\Psi_{A T I, 0}=\arg \left(V_{2} \cdot V_{1}^{*}\right)=k_{0} B_{a} \sin \phi .
$$

This phase is deterministic, and it can be removed from the interferometric data using an external DEM, the transmitter trajectory and the receivers positions.

\subsection{Moving target}

If a target is moving at a constant velocity during the SAR integration time, it produces a bistatic range derivative, $\dot{R}_{b}$, which is constant. The resulting linear phase term will result in a shift of the apparent azimuth position of the target. This shift can be expressed in time as

$$
\tau_{a}=-\frac{\dot{R}_{b}}{\lambda_{0} K_{a}}=-\frac{R_{t 0} \dot{R}_{b}}{v_{x}^{2}}
$$

and it affects all the ATI channels in the same way. If the theoretical ATI phase related to the angular position is substracted to the ATI phase, the residual phase will be

$$
\begin{aligned}
\Psi_{\text {res }} & =\Psi_{A T I}-\Psi_{A T I, 0} \\
& =-k_{0} B_{a} \frac{t_{a} v_{x}}{R_{r 0}}+k_{0} B_{a} \frac{\left(t_{a}-\tau_{a}\right) v_{x}}{R_{r 0}} .
\end{aligned}
$$

Using (3), $\Psi_{\text {res }}$ can be expressed as

$$
\Psi_{\text {res }}=k_{0} \dot{R}_{b} \tau_{A T I}
$$

which is the expected expression depending on the temporal lag and the bistatic range variation due to a moving target. If the transmitter and the receiver are on the same side of the observed scene, which is our case, the measured velocity in the bistatic range direction, $v_{b}$ can be related with $\dot{R}_{b}$ as

$$
v_{b}=\frac{\dot{R}_{b}}{2} .
$$

The maximum unambiguous velocity that can be measured is $v_{b, \max }=\frac{\lambda}{4 \tau_{A T I}}$ and it depends on $R_{r}$. For orbitalground bistatic geometries, even for small along-track baselines, it results in small unambiguous velocities, or in other words, the system is very sensitive to velocity. It has to be noticed that the formulation employed assumes that all the resolution cell is moving. This can be a valid assumption for a sea surface or if there is only one moving target with a high Signal to Clutter Ratio (SCR), thus the clutter can be ignored. For other scenarios, there will be the contribution of the moving target (or targets) and the static clutter. In order to estimate more than one contribution, multiple baselines and spectral estimation algorithms have to be used.

\subsection{Multi Along Track Baseline Implementation}

If multiple receivers performing a set of baselines are available, it is possible to estimate more than one moving contribution for each resolution cell. Assuming that $\mathrm{N}$ images are obtained, the first step is to remove the deterministic ATI phase with respect a master image. Once, this systematic phase component has been removed, it is possible to establish a signal multi along track baseline model analogous to the one used in SAR Tomography. The measured data vector $y$, for range-azimuth position, is

$$
y=\sum_{i=1}^{N_{s}} \sqrt{\tau_{i}} x_{i} \odot a\left(v_{b}^{i}\right)+n
$$

where $\odot$ is the Hadamard product, $x_{i}, a\left(v_{b}^{i}\right)$ and $n$ are $\mathrm{N}$ dimensional vectors representing the multiplicative noise, the steering vector related to the velocity of the i-th source in the bistatic range direction and the thermal noise. $N_{s}$ is the number of relevant sources in the resolution cell and $\tau_{i}$ is the reflectivity of the i-th source. In this case, the steering vector is related to $v_{b}$ as

$$
a\left(v_{b}\right)=\left[1, e^{j k_{0} 2 v_{b} \tau_{A T I_{1}}}, \ldots, e^{j 2 k_{0} v_{b} \tau_{A T I_{N-1}}}\right] .
$$

The $\tau_{A T I_{i}}$ is related to the $\mathrm{i}$-th along-track baseline $B_{a_{i}}$. The number of sources that can be estimated depend on the number of independent baselines as

$$
N_{s} \leq N_{B_{a}}
$$

Many spectral estimators can be employed to retrieve the reflectivity and the velocity in the bistatic range direction of each source like Capon, the classical Beamforming (BF), MUSIC, Non Least Squares (NLS), ... 


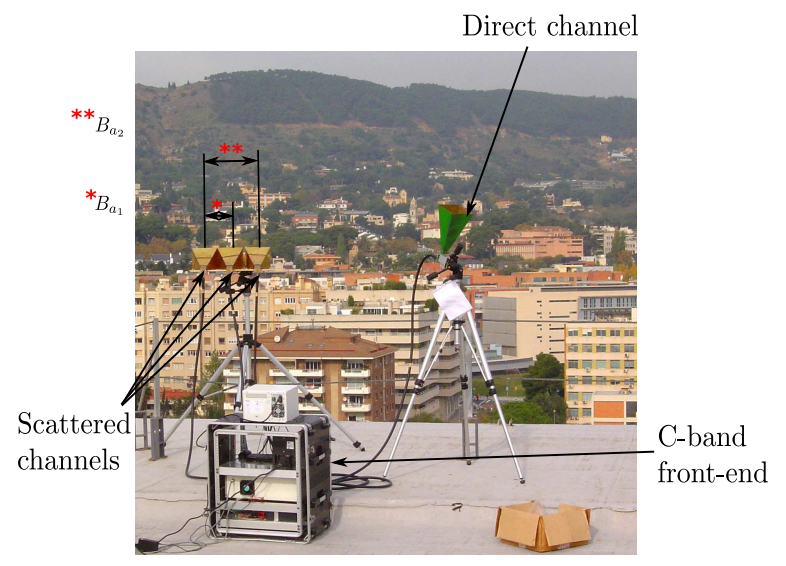

Fig. 2. SABRINA set-up for a bistatic multibaseline alongtrack acquisition.

\section{EXPERIMENTAL RESULTS}

\subsection{Experimental set-up}

Experiments have been carried out using UPC's C-band SABRINA system and ESA's ENVISAT satellite. A description and discussion of an earlier 2-channel version of the system can be found in [2]. Now, the system has 4 channels.

The absence of a dedicated link between the transmitter and the receiver local oscillators results in the necessity of using a direct signal for PRF recovery and phase synchronization [1]. This direct signal is obtained using one dedicated channel, with an antenna pointing directly to the satellite. The other 3 channels are placed performing two independent along-track baselines, $B_{a_{1}}$ and $B_{a_{2}}$ of 18 and $37 \mathrm{~cm}$ respectively. Figure 2 shows the set-up of the acquisition system for this experiments. SABRINA was placed at the top of a 54 meter tall building at UPC's campus.

In order to have a controlled moving target, a Bistatic Active Radar Calibrator (BARC) has been used. The BARC consists in an antenna pointed to the satellite, an amplification chain and a transmitter antenna pointed where SABRINA is located. The BARC was attached to a linear unit and its motion was synchronized with the satellite pass. The BARC was moving at a constant velocity of $20 \mathrm{~cm} / \mathrm{sec}$ towards the receiver. The velocity vector projected over the bistatic range where the BARC was placed was $v_{b}=15.1 \mathrm{~cm} / \mathrm{s}$. Figure 3 shows a picture of the BARC attached to the linear unit.

\subsection{Results}

This section shows the geocoded results using the $18 \mathrm{~cm}$ baseline for the points with a SNR higher than $15 \mathrm{~dB}$ and the MB results. For the latter, the obtained amplitude versus velocity profile for two different resolution cells with different inversion methods is presented. Figure 4 shows the geocoded received power, the BARC can be easily identified as it presents

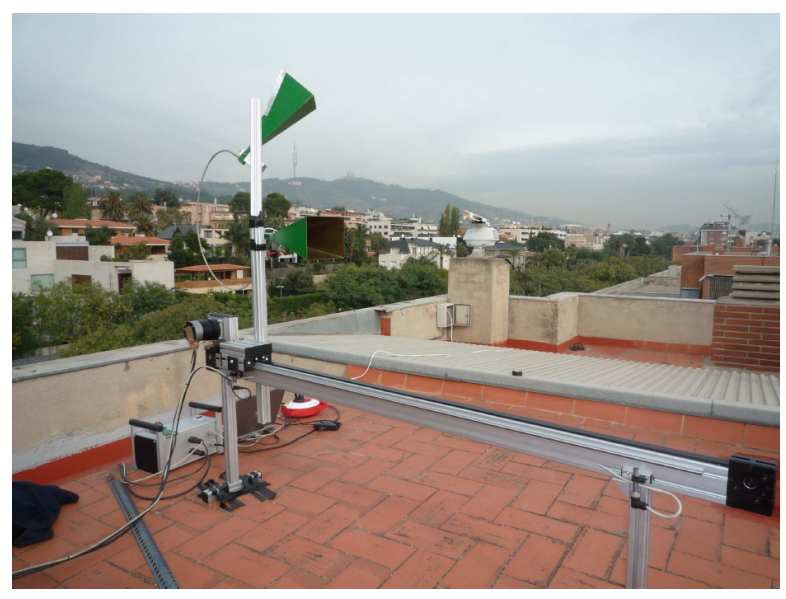

Fig. 3. Controlled moving target consisting on a BARC attached to a linear unit.

a high SCR. In addition, its high SCR allows to estimate its velocity with a single baseline. The ATI phase is illustrated in Figure 5, it presents fringes in the ortogonal direction to the receiver range. Once the deterministic term of the phase has been removed, the phase can be converted to velocity.

As it can be seen in Figure 6, the major part of the selected points are static, they are represented in green. There is a red spot where the BARC is located related. The measured velocity in the bistatic range direction for the BARC is $v_{b}=$ $15.0 \mathrm{~cm} / \mathrm{s}$, very close to the expected value, $15.1 \mathrm{~cm} / \mathrm{s}$.

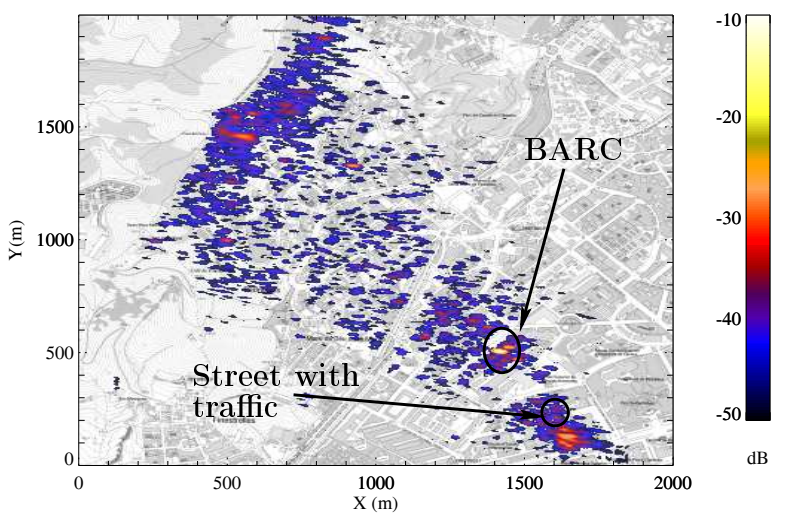

Fig. 4. Geocoded received power.

Capon, MUSIC, BF and NLS have been used as inversion methods to retrieve the velocities with the multibaseline approach. Figure 7 shows the results from the multibaseline approach for the BARC, 7(a), and a street with some traffic, 7(b), using Capon, MUSIC assuming $1, N_{s}=1$, and 2, $N_{s}=2$, targets, BF and NLS as inversion methods. Taking a look to Figure 7(a), it can be said that BF finds the solution, but it presents high sidelobes, due to the irregular base- 

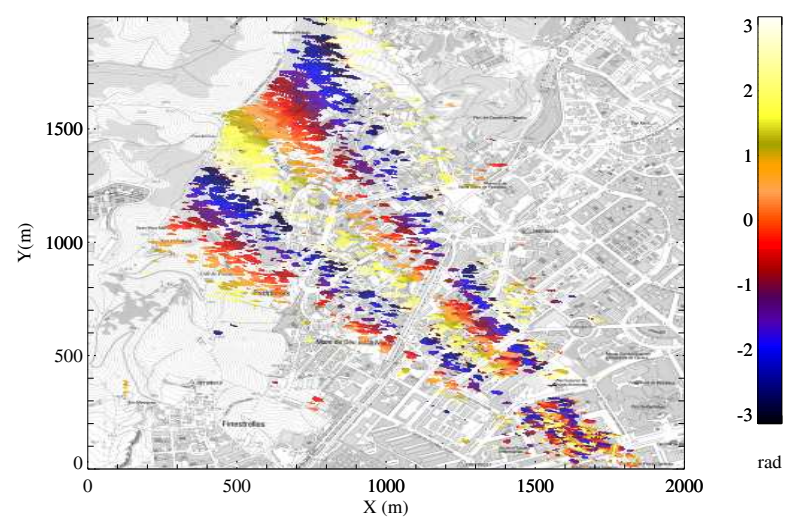

Fig. 5. Geocoded raw interferometric phase.

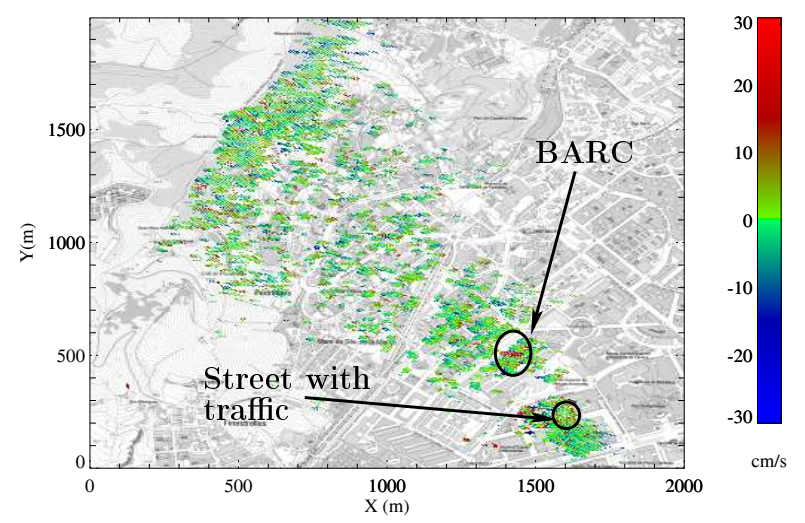

Fig. 6. Geocoded $v_{b}$.

line sampling. Capon also gives the correct solution wit no secondary lobes. MUSIC performance is good if the assumption of number of targets is correct. Finally, the NLS method retrieve the velocity with high precission. Focusing in the results for the pixel with some traffic, the expected behaviour is to observe two main velocity contributions, the static clutter and a moving target. It is extremely likely that the velocity of the car is over the non-ambiguous one, thus the system can be only used for GMTI. In this case, BF shows a bad performance, it only retrieves one contribution due to the car. Capon retrieves both of them but with poor precission. MUSIC with $N_{s}=2$ and NLS produce similar results, retrieving both contributions, clutter and moving target, wiht good precission. Finally, MUSIC assuming only onet target finds a single contribution with a velocity different to the previous ones.

\section{CONCLUSIONS}

Along-track interferometry is a valid tool for a bistatic or multistatic configurations. It would be an useful application for

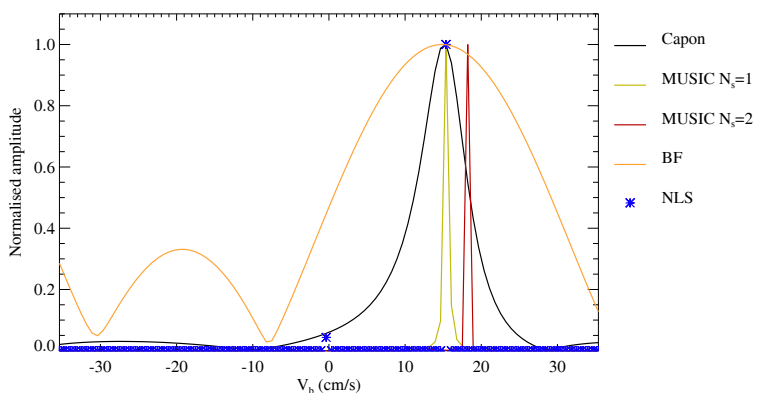

(a)

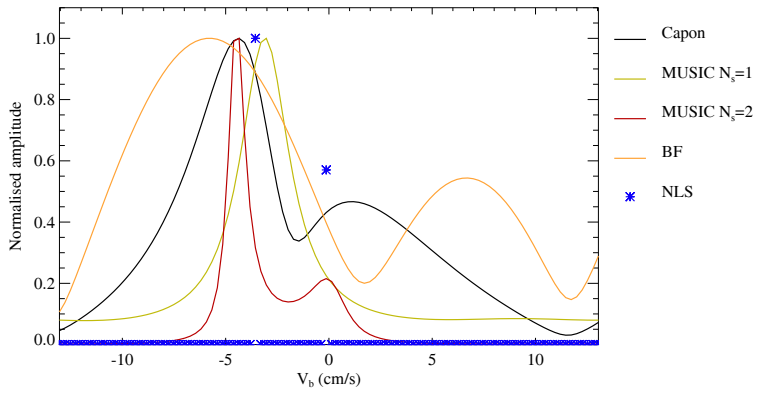

(b)

Fig. 7. Results from the MB approach for the BARC, Figure 7(a), and a street with some traffic, 7(b), using different inversion methods.

sea surface currents estimation or for GMTI. In this paper, the theory about bistatic along-track interferometry has been presented and it has been extended to the case of multiple receivers. Results over real data with a controlled moving target have been presented. These results show a good agreement between the expected velocity for the target and the measured one, thus, the technique has been validated. MB approaches can be usefull to distinguish between two or more velocity contributions in the same resolution cell.

\section{REFERENCES}

[1] P. Lopez-Dekker, J.J. Mallorqui, P. Serra-Morales, and J. Sanz-Marcos. Phase synchronization and doppler centroid estimation in fixed receiver bistatic SAR systems. Geoscience and Remote Sensing, IEEE Transactions on, 46(11):3459-3471, 2008.

[2] J. Sanz-Marcos, P. Lopez-Dekker, J.J. Mallorqui, A. Aguasca, and P. Prats. SABRINA: a SAR bistatic receiver for interferometric applications. Geoscience and Remote Sensing Letters, IEEE, 4(2):307-311, 2007. 